\title{
A comparative and comprehensive study of prediction of Parkinson's disease
}

\author{
N. Prasath ${ }^{1}$, Vigneshwaran Pandi ${ }^{2}$, Sindhuja Manickavasagam ${ }^{3}$, Prabu Ramadoss ${ }^{4}$ \\ ${ }^{1,2}$ Department of Computer Science and Engineering, SRM Institute of Science and Technology, Chennai, India \\ ${ }^{3}$ Department of Information Technology, Rajalakshmi Engineering College, Chennai, India \\ ${ }^{4}$ Senior Solution Architect, Dassault Systemes Austrila Pty Ltd, Western Australia, Austrila
}

\begin{tabular}{l} 
Article Info \\
\hline Article history: \\
Received May 19, 2021 \\
Revised Jul 30, 2021 \\
Accepted Aug 9, 2021 \\
\hline
\end{tabular}

\section{Keywords:}

Artificial intelligence

Classification

Kernel based extreme learning

Machine learning

Parkinson's disease

Prediction

\begin{abstract}
Parkinson's disease (PD) is a form of neurodegenerative disease that is caused the progressive weakening of dopaminergic nerve cells that affects a large number of people around the world. The recent treatment methods principally depend upon the experimental data resulting from assessment balances and patients' journals that take varied boundaries with reference to legitimacy, inter-rater inconsistency, and incessant monitoring. Nowadays various computational Intelligence techniques are utilized in predicting an accuracy of PD and these techniques are widely applied to form the acceptable decision accurately. In this paper an in-depth review was administered on various techniques proposed by numerous researchers. A replacement system must be proposed which uses DL techniques and considers other attributes of paralysis agitans which can improve the prediction and be an advancement within the medical field. It has been observed that many researches have been done in identifying the PD yet there is a need of suitable method or algorithm to improve the prediction of PD which helps the clinical management. In order to increase the precision approaches involving movements, facial expression and other attributes also be considered for evaluation, since most of the methods have used speech as a major attribute.
\end{abstract}

This is an open access article under the CC BY-SA license.

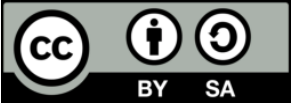

\section{Corresponding Author:}

Vigneshwaran Pandi

Department of Computer Science and Engineering

SRM Institute of Science and Technology

Kattankulathur-603 203, Chennai, India

Email: vignenesp@srmist.edu.in Øst, Denmark

Email: corresp-author@mail.com

\section{INTRODUCTION}

Parkinson's disease may be a liberal neurodegenerative illness considered by inactive tremor, akinesia, rigidity, bradykinesia and postural unpredictability, produced by discerning collapse of dopaminergic nerve cell inside the nucleus niger, leading to lower dopamine levels within the brain. Mutations within the glucocerebrosidase gene constitute the only largest risk factor for the event of sporadic paralysis agitans. An environmental factor for Parkinson's includes drinking ground water and consuming manganese. Another common divisor for Parkinson's is identified as lewy bodies. Lewy bodies (protein clumps) cover cells in the brain, interfering with their functions. Cramped handwriting, uncontrollable movements while sleeping, limb stiffness, voice changes, rigid countenance or masking, and stooped posture are all early signs of PD. Also, disturbance of memory and thinking ability may be a non-motor symptom related to Parkinson's. Many of us with Parkinson's disease feel distracted or disorganized, or have difficulty 
planning and completing tasks. To begin, based on the assessment of the relationship between GBA mutation status and neuropsychological outcomes, the status of GBA mutation is an independent risk factor for cognitive impairment in patients with PD, clinical dementia rating (CDR), and clinical diagnoses [1]. Patients of Ashkenazi-Jewish origin with Parkinson's disease were also screened for seven founder GBA mutations, and the findings revealed that for both moderate and severe heterozygous individuals, the risk and age at onset (AAO) of PD were increased. GBA mutations were differentially affected, which had important implications for guidance [2]. A multitask diffusion adaptation strategy with mixture weights, accompanied by simulation of connectivity in the brain and applying intensities, is used to distinguish between brain functions ie., functions is normal or abrormal. The findings show that multitask learning approaches are often used to improve brain communication patterns [3]. Using SVM (for calculating training set error minimization and margin maximisation) to accurately predict Parkinson's disease, assisted bacterial foraging optimization (BFO) was developed. This achieved optimal generalisation ability while preventing overfitting and defined the key parameters for effective prediction [4]. Asynchronous detection supported deep brain stimulation was wont to monitor the patient's hand movements. The results produce a difference between the working of the left and right hemispheres [5]. Additionally, the wearable sensor was wont to record the daily activity and this was mainly utilized in a life style environment. For physical activities, the experiment yields time quantity analyses [6]. A common method of evaluating postural instability in PD patients is by conducting pull test (a tug on the patient's shoulders). It takes ten factors into account in order to shift the centre of gravity away from neutral. The findings were used to build a ROC graph and to categorise patients [7]. To supply patients with effective exercises and to enhance their posture stability SBS rehabilitation of dynamic weight shifting mechanisms was used. It detects the postures and tent of patients during their course and provides feedback to enhance the steadiness [8].

\section{RELATED WORKS}

Hadjahmadi and Askari [9] proposed a classification based emotionally supportive network for determination of Parkinson's infection. Parkinson's unwellness, the problem conjointly ordinarily causes a speed or freeze of development. Regression, support vector machines (SVM), classification and regression trees (CART), bayesian networks, and artificial neural networks were all used in the proposed framework (ANN). Ali Saad et al. [10] proposed a Bayesian belief network for detecting infected patients and customs a motion dataset obtained from real Parkinson's disease patients who were walking at the time and had real change times. Each document was created using a framework that included estimating facts from the three sensors in order to determine whether or not climate freezing of gait (FoG) occurred. Amit et al. [11] investigated a system for grouping and sorting Parkinson's patients based on their postural response using an L2 standard measurement linked to support vector machines and 24 patients were esteemed when medicine. Every quiet languished following investigation convention over the assessment of their property stability: beginning, eyes open on force stage and second, eyes open on foam put on force stage (FO). Sharma and Giri. [12] proposed fake neural organization, design acknowledgment and backing vector machine. It is acclimated provision the specialists inside the assignment of Parkinson unwellness. Tiwari [13] proposed a base excess greatest pertinence include choice calculations want to choose the premier significant component among every one of the highlights to foresee the Parkinson sicknesses. The changed AI based techniques like sacking, boosting, arbitrary backwoods, revolution woods, irregular subspace, svm, multifacet perceptron, and choice tree-based strategies are wont to anticipate loss of motion agitans. The base excess greatest pertinence highlights determination calculations have acclimated select the premier significant element among every one of the highlights from the discourse enunciation trouble manifestations of Parkinson's sickness influenced individual to anticipate loss of motion agitans. Tsanas et al. [14] proposed how precisely the new algorithms are used to favor PD subjects from healthy controls. From continued vowels, there are a total of 132 dysphonia steps. Four feature selection algorithms are used to pick and SVM and random forest are the two classifiers, that are used to map to a binary classification answer. This method makes use of an existing database that includes 263 samples are collected from 43 subjects. The new dysphonia measures with ten dysphonia features outperformed well and achieved the accuracy of $99 \%$ in classification.

Chen et al. [15] developed two methodologies, the Nested-RF and Nested-SVM classifiers, can search the model's ideal boundaries naturally. Five datasets of malignant growth (brain cancer, carcinoma, DLBCL, leukaemia, and prostate cancer) and one of the proposed classifiers' output was evaluated using a dataset of illnesses. Loss of motion agitans characterization, the nested-SVM classifier showed better execution with the exactness up than ninety \{three\} that was two-hundredth an incredible outcome from elective three classifiers. Azad et al. [16], Classical choice tree, ID3, and choice stumps, a name given, are used for preparing and assessing the feasibility of many symptoms that contribute to Parkinson's disease, 
such as tremors in the legs, arms, and hands, impaired discourse clarity, and developmental issues. For a powerful dataset to differentiate healthy persons from those with Parkinson's illness, Bouchikhi et al. [17] proposed artificial neural networks (ANN), Data mining neural, regression, and decision tree. for successfully finding datasets to isolate solid individuals and lack of motion agitans, artificial neural networks (ANN), knowledge handling neural, regression, and decision trees were used. The nested-random forest (nested-RF) and nested-support vector machine (nested-SVM) classifiers were introduced by Chen et al. [18] for the prediction of five malignancy datasets (cerebrum disease, carcinoma, DLBCL, leukaemia, prostate malignant growth) and one sickness dataset (Parkinson's).

Chen and Lin [19] introduced two methodologies, the nested-RF classifier and the Nested-SVM classifier, that can search for the model's ideal boundaries naturally. We apply these classifiers to five malignant growths (mind malignant growth, DLBCL, prostate malignant growth, colon malignant growth, and leukaemia) as well as Parkinson's disease to see how successful they are. Using the ANOVA consistency determination technique, the major qualities were selected from those with a $\mathrm{P}$ value of less than 0.05 . The suggested Nested-SVM classifier is proven to be the best after examining the characterization data obtained from four different classifiers. The Nested-SVM classifier outperforms the other three classifiers by around $2-5 \%$ in terms of grouping execution (exactness, affectability, and explicitness) when applied to five malignancy datasets. Chen et al. [20] suggested an FKNN-based system in relation to support vector machines (SVM)-based methodologies for a dataset containing 31 biomedical voice estimations from 23 Parkinson's disease patients. The FKNN-based framework's highest order precision (96.07 percent) obtained using a 10-crease cross approval strategy will ensure a solid analytic model for Parkinson infection position. The proposed structure by Cho et al. [21] combines a calculation joining head part investigation principal components analysis (PCA) with direct linear discriminant analysis (LDA). It suggested a step-by-step examination framework for locating a walk example of Parkinson's disease misuse of computer vision. Farhad S G and Peyman M. [22] introduced an MLP, RBF, and ANN-based method to distinguish between clinical factors of tests $(\mathrm{N}=195)$. MLP and RBF characterization exactness for the knowledge set was 93.22 percent and 86.44 percent, respectively. To predict behavior related to quake starting, Wu D et al. [23] proposed an outspread premise work neural organization radial basis functional neural network (RBFNN) in light of molecule swarm advancement particle swarm optimizer (PSO) and head part investigation with local field potential (LFP) information recorded by means of the incitement anodes to foresee movement identified with quake beginning. LFPs from the subthalamic core (STN) got through profound cerebrum cathodes embedded in a Parkinson patient are utilized to prepare the organization. Eelectromyographic (EMG) signals from the patient's lower arm were captured and in conjunction with LFPs to control quake events, which are then compared to the organization's display. It has been discovered that precise position accuracy of up to 89 percent is possible. Execution correlations between a standard RBFNN and an RBFNN based on PSO have also been performed, showing a slight reduction in execution but a large reduction in computational overhead. To distinguish solid individuals from those with Parkinson infection, Hazan et al. [24] proposed the subtractive clustering features in a kernel-based extreme learning machine weighting methodology (CFW-KELM).

Hung Nguyen et al. [25] developed a method to handle input from the inertial estimation unit in order to identify and fragment unstructured ADL in persons with Parkinson's disease (PD) living in the community. Using the suggested technique, 1610 ADL occurrences were precisely located within 350 milliseconds of the "best quality standard" manual division by nine local older individuals with PD in a recreated free-living environment with 90\% precision (affectability $=90.8$ percent, explicitness $=97.8$ percent). People with Parkinson's disease may spontaneously discriminate and segment ADL in a free-living setting using the suggested approach, according to these findings. Rustempasic et al. [26] suggested a naturally AI approach and established the Parkinson infection for the benefit of the individual's discourse/voice. The developer used a fluffy $\mathrm{C}$-implies bunch and example recognition-based approach to distinguish between sound and parkinson's disease-affected people. To distinguish loss of motion agitans from sound topics, Rusz et al. [27] proposes to find the simplest blend of estimations, an implemented support vector machine was suggested. This system prompts an eighty-five percent general grouping execution. In fact, we've discovered ties between correspondence and explanation proportions and bradykinesia and unbending nature in Parkinson's disease. Kapoor and Sharma [28] suggested that MFCC and VQ acknowledge debate. The MFCC employs discourse examination outlines in relation to the recurrence space, and the Vector division was chosen as the codebook with the least twisting. The twenty phonations used in daily conversation and in patients with Parkinson's disease. In classifiers 90th and 95th, respectively, In the customary voice and voice of Parkinson's disorder rate, vector division results with codebook. The discretization strategy, help vector machines, C4.5, k-closest neighbours technique, and Naive Bayes more tasteful techniques that are wont to group the dataset were suggested by Kaya et al. [29]. The dataset was organised using discretized and non-discretized judgments, implying that discretization can be 
used to attribute loss of motion agitans. Using each of the 22 highlights and a parallel choice class (' 0 ' is solid and ' 1 ' is IPD choice class), Revett et al. [30] proposed jitter, sparkle, important, music/commotion proportions, enlightening measurements, and correlational elements (non-straight powerful examination). A legendary animal and disarray structure were created to appear at the precision of the grouping technique, and the research partners degreed instructing attempt was characterised. The probability of exactness is 100 percent. Khemphila and Boonjing [31] suggested a multi-layer perceptron (MLP) with a Back-Propagation learning calculation that could be used to evaluate Parkinson's disease effectively (PD). The specialist's knowledge and expertise completed the discovery. However, there have been cases of incorrect assignment and treatment reported by territory units. Patients' area has demanded that a range of tests be needed for assignment. In certain cases, not all of the tests are helpful in determining the cause of an illness. The patients' assignments were acclimated by the engineered neural organisations zone. Kihel and Benyettou [32] suggested clonclas and probabilistic neural network (PNN) were proposed as tools for distinguishing between solid and Parkinson's infection patients (PWD). We strive to steal accommodating properties like programmed acknowledgment, memorisation, and transformation by taking inspiration from usual invulnerable frameworks. Clonclas was fascinated by the algorithmic standards that were developed based on the measurement of training medication.

Rouhollahi et al. [33] proposed a closed-loop control framework to invigorate two zones of BG all the while. The oddity of the proposed regulator structure is twofold: initial an AFEL/PSF regulator, and second concurrent incitement of GPi and STN. To convey reasonable stimulatory control sign to the cerebrum, closed-loop control framework with criticism is utilized from hand quake. Thus, quake has direct connection to stimulatory energy force. As a result, the stimulatory energy capacity is drastically decreased. We were able to reduce the computational time and increase the information set by combining fluffy entropybased component choice with a closeness classifier, as proposed by Luukka [34]. To gather data, a variety of drug voice estimates from healthy people and people with Parkinson's disease were used (PD). For the Parkinson infection dataset, Ma et al. [35] suggested a completely new crossover technique called kernelbased extreme learning machine with subtractive clustering features weighting (SCFWKELM), which basically beats SVM, KNN, and outrageous learning machine (ELM) approaches. Can [36] suggested an equal distributed neural organisation of couple of hidden layers, assisted in the aim to use of separating and a lion's share casting a ballot system to identify people with traditional vocal signs and Parkinson's disease symptoms. Caglar et al. [37] proposed using ANNs multilayer perceptron (MLP) and radial basis function (RBF) Networks, as well as adaptive neuro-fuzzy classifier (ANFC) with phonetic fences to distinguish solid people with individual palladium. The least challenging acknowledgment findings came from a versatile Neuro-Fuzzy Classifier with etymological help. For grouping solid and Parkinson's patients, Shahbakhi et al. [38] used a genetic algorithm (GA) and a support vector machine (SVM). F0 (major recurrence or pitch), jitter, gleam, and commotion to sounds proportion, that are all essential factors for voice signal, were all upheld by voice flags. Chen and Lin [19] developed dual methodologies, Nested-RF and Nested-SVM classifiers, that can look for the model's ideal boundaries in a natural way. To evaluate the presentation of the proposed classifiers, five datasets of malignancy (mind disorder, colon malignancy, DLBCL, leukaemia, and prostate disease) and one dataset of illness (Parkinson's) were used. In the grouping of Parkinson's disease, the Nested-SVM classifier had a stronger presentation with a precision of more than ninety-three, which was two hundredth an incredible result from the elective three classifiers.

Gullible Bayes, channel determination naive Bayes (FSNB), naive Bayes relation based with highlight subset option strategy (CFS-NB), and backing vector machines were proposed by Morales et al. [39]. (SVM). Ramani and Sivagami [40] suggested a number of data mining methods, including SVM, KNN, Random tree, partial least square regression (PLS), and so on, to predict dataset medication voice estimations from 31 individuals, 23 of whom had Parkinson's disease (PD). The calculations for higher characterization purpose were added to the sifting, with the most modest number of characteristics for which the better order was selected and achieved. Nivedita et al. [41] suggested a fake neural organisation (ANN) with backpropagation to group neurodegenerative problems with side effects in a consistent manner. There are six distinct types of clinical side effects associated with neurodegenerative diseases. Problems such as Memory, communication, personality changes, odd behaviours, lack of willful control, and standard medical problems are all common. PC assisted determination (CADx) systems were introduced by Ozcift and Gulten [42] to increase precision. To polygenic uncertainty, heart, and Parkinson's datasets, transform woods (RF) aggregate classifiers of thirty AI algorithmic guidelines relation based generally highlight decision (CFS) calculation and Rotation woodland forecast. Prashanth et al. [43] proposed support vector machine (SVM) and factory loss data from the 40-item University of Pennsylvania Smell Identification Test (UPSIT) and sleep behaviour data from the rapid eye development rest behavior disorder screening structure (RBDSQ) from the Parkinson's progression marker's initiative (PPMI) data collection were used to create arrangement tree strategies. 
Przybyszewski [44] suggested the development lines in the stage space are plotted reflexive saccades estimations and arrangements to forecast singular patients and little understanding common critical measure impacts as changes of the necessary hip x-points as a work of the left hip point changes all through three stages of stable strolling and discovered different forms of attractor changes as the left hip point changes. To predict medicine voice estimates from 31 participants, 23 of whom had lost motion agitans, Das [45] suggested neural organizations, knowledge preparation, and neural networks. A relative report on the loss of motion agitans informational index was generated using neural investigation, multivariate analysis, and option trees. The procedure was assigned Parkinson's disease upheld the SAS code, according to the characterization. Alcalay [46] involved CORE-PD participants who were tested for PARKIN, LRRK2, and GBA transformations were given a neuropsychological battery, while those who were tested for PARKIN, LRRK2, and GBA transformations were given a smell identification test (UPSIT) by the University of Pennsylvania. There were 33 GBA transition transporters and 60 noncarriers with some inherited transformation among the group's members. Essential tests were carried out on 26 GBA heterozygous shift transporters without extra transformations and 39 noncarriers with age and PD terms coordinated. Changed z scores of individual neuropsychological assessments were used to establish five psychological areas: psychomotor speed, consideration, memory, visuospatial ability, and leader work. Clinical analyses (normal, mild intellectual impairment [MCI], dementia) were classified as incognizant in terms of genotype based on neuropsychological execution and utilitarian hindrance as measured by the clinical dementia rating (CDR) ranking. GBA transition status was investigated in relation to neuropsychological execution, CDR, and clinical decisions. Armañanzas et al. [1] suggested five different grouping criteria based on a covering highlight option plan that is capable of predicting all of the classification variables with an accuracy range of 72-92 percent. Furthermore, grouping into the three main seriousness classifications (gentle, moderate, and extreme) was incorporated into polarity problems, in which paired classifiers outperform single classifiers and choose different subsets of non-engine manifestations.

The expectation maximization (EM) calculation was proposed by Saad et al. [47], who proposed a method for learning a probabilistic graphical model by itself bayesian belief network (BBN) is a network of Bayesian beliefs. Voice and penmanship are used to forecast a blended procurement arrangement with electronic pen and discourse signals. Based on the investigation of voice and penmanship, this paper predicts collection. Salhi et al. [3] proposed a method for extracting an element vector from discourse tests using wavelet analysis, after that, a multilayer neural network (MNN), a three-layer feed-forward network with sigmoid actuation, and a back propagation calculation (BPA) classifier were used. For reverberation images, Salvatore et al. [48] proposed to predict individual differential conclusions of Parkinson's disease, a controlled AI calculation based on principal components analysis as a highlight extraction method and support vector machines was used (PD) and progressive supranuclear palsy (PSP) MRI dataset. The proposed arrangement of obsessive voice from typical voice by Sellam and Jagadeesan [49] uses support vector machine (SVM) and radial basis functional neural network (RBFNN) in the implementation of RBFNN. Adolescent voices, both regular and neurotic, are accustomed to training and researching classifiers. The discourse signal was then dissected to identify acoustic boundaries including Signal Energy, pitch, and formant frequencies. Stemmer [50] suggested an SVM and Correlation-based characterization of Parkinson's disease impacted an individual's discourse/speech. Parkinson's disease was recognised using verbalization, voice, and prosodic assessments. Tsanas et al. [51] suggested sign approach using nonlinear method with a large dataset (dataset is voice/discourse reported without the need for a doctor in the clinical) distributed over a large area to measure discourse signal. It checked the perceivability of successive, far away, real, precise UPDRS telemonitoring based on self-managed discourse tests and conducted abuse nonlinear relapse and order recipe. Yang et al. [52] proposed three relative investigations are intended to assess the presentation of MegaSNPHunter. The primary research relies on manufactured data obtained from epistasis models. The following one is focused on a genome-wide analysis of Parkinson's disease (information procured by utilising Illumina HumanHap300 SNP chips). The third one selects the rheumatoid joint inflammation concentrate from the wellcome trust case control consortium using the Affymetrix GeneChip 500K mapping array collection (WTCCC). MegaSNPHunter outperforms the competition and announces a slew of planned collaborations for the two genuine tests.

Cai et al. [53] suggested that an optimal support vector machine (SVM) be set up in view of bacterial searching improvement (BFO) to effectively predict PD. On the basis of a PD informational selection based on vocal estimations, the feasibility of the proposed technique, BFO-SVM, was accepted. The proposed technique and two of the most commonly used boundary advancement techniques were compared to an SVM based on the structure quest strategy and an SVM based on molecule swarm enhancement. Furthermore, to increase forecast accuracy, the help highlight determination was used before the BFO-SVM technique, resulting in the RF-BFO-SVM being proposed. The results showed that the system worked extremely well in terms of arrangement execution, with an average order exactness of 97.42 percent. Mandal 
and Sairam [54] used a robust inference method, researchers suggested an improved diagnosis prediction accuracy for Parkinson's disease (PD) to avoid patient delays and misdiagnosis. The proposed system used metrics for determining prediction accuracy, such as specificity, sensitivity, accuracy, and other observable parameters. Using linear logistic regression and sparse multinomial logistic regression, this proposed scheme achieved the highest accuracy of 100 percent, 0.983 is sensitivity, and Specificity: 0996. The sparse multinomial logistic regression, rotation forest ensemble with support vector machines and principal components analysis, artificial neural networks, and boosting methods are all used in the robust inference system.

Pahuja and Nagabhushan. [55] commented that on the benchmark (voice) dataset, they proposed three types of classifiers, namely multilayer perceptron, support vector machine, and K-nearest neighbour, were discussed to compare and determine which of these classifiers is the most effective and accurate for PD classification. The main challenge of prediction of PD is identifying the most appropriate classification algorithm on local dataset. Rastegar et al. [56] proposed a used serum samples from a clinically wellcharacterized longitudinally followed Michael J Fox Foundation cohort of PD patients with and without the common leucine-rich repeat kinase 2 (LRRK2) G2019S mutation to suggest a prediction of PD progression. The best prediction models for the motor symptom severity scales, with NRMSE of 0.1123 for the Hoehn and Yahr scale and 0.1193 for the unified Parkinson's disease rating scale part three, were found using the normalised root mean square error (NRMSE) as a measure of efficiency (UPDRS III). To predict 2-year longitudinal clinical outcomes, the proposed model used predictive modelling with machine learning, with the elastic-net53 and random forest54 algorithms being trained on a combination of clinical variables, inflammatory cytokine measurements, and demographic variables (age and gender). Elastic net is a linear regression technique that minimises overfitting by using both $\mathrm{L} 1$ and $\mathrm{L} 2$ regularizations. Mathur et al. [57] proposed a various machine learning algorithm to improve the performance of datasets and early prediction of disease at right time. The accuracy obtained from the combined effect of the KNN algorithm with ANN is greater than that obtained from other algorithms, according to the experimental results. Ulagamuthalvi et al. [58] proposed a method provide the high accuracy of prediction for the Parkinson's disease dataset using machine learning algorithms. The proposed system used two machine learning algorithms which are logistic regression and XGBoost. The maximum classification rate is achieved by XGBoost than LR with an accuracy $96 \%$, whereas LR achieved only 79\% accuracy. Ulagamuthalvi [59] given We were able to meet the large dataset size requirements by using a supervised data collection method that enabled us to generate detailed annotations in one-minute intervals. To our knowledge, no other large-scale collection of expert annotations on a one-minute basis has been published. Ulagamuthalvi [60] have presented in the field of computer vision, classification is a crucial role. Image classification is the method of categorizing images into one of many predefined groups, which include image sensors, image pre-processing, object detection, object segmentation, feature extraction, and object classification. For image classification, a variety of techniques have been developed. The highest concentration is on using various classifiers combined with several segmentation algorithms for detection of tumor using image processing.

Viswanathan and Naib [61] have proposed as Performance parameters used by authors are true positive, true negative and accuracy. Authors make use of various semi-supervised classifiers for intrusion detection. All classifiers used NSL KDD dataset for intrusion detection. Mozhdehfarahbakhsh et al. [62] presented the back propagation approach compares normality and abnormality using a feedback-based approach. The derived feature sequences of regular and abnormal walking, as well as the three classes A, B, C, D normal, Parkinson gait, Hemiplegic gait, and Neuropathic gait data sets, were compared with the normal data set during the training process. Mozhdehfarahbakhsh et al. [63] proposed a Convolutional Neural Network and MRI based deep learning model to predict the Parkinson 's disease and its stages. In Parkinson's disorder always a challenging to identify its stages and its progression. Based on the technical advancements in Artificial Intelligence, supervised and unsupervised machine learning methods are used on clinical and paraclinical datasets to accurately diagnose PD, identify its stage and predict. The MRI dataset is used in the proposed model to effectively predict the PD and identify its stages. The proposed method produced $94 \%$ of accuracy to distinguish the stages. Shahid and Singhn [64] proposed automated ML pipeline model to make improved multi-omic forecasts of PD. The multimodel information is utilized in robotized ML framework to tune the exhibition. The underlying model showed a territory under the bend (AUC) of $89.72 \%$ for the analysis of PD. The tuned model was then tried for approval on outer information (PDBP, AUC 85.03\%). Enhancing limits for order, expanded the conclusion forecast precision (adjusted exactness) and different measurements. Joining information modalities beats the single biomarker worldview. The model created improves illness hazard expectation. The proposed framework develops the quality articulation networks for the up-and-coming age of genomics-inferred mediations. Our mechanized ML approach permits complex prescient models to be reproducible and available to the local area. Shahid and Singh [65] proposed a PCA based profound neural organization (DNN) model to foresee Motor-UPDRS and 
Total-UPDRS in Parkinson's illness (PD) movement. The model utilized the decreased info highlight space of Parkinson's telemonitoring dataset for checking the movement. PCA was utilized to extricate the highlights from the entire dataset that incorporate age and sex of the PD patients. Clinically, the age and sex influence the UPDRS score. The model's expectation precision is estimated by wellness boundaries, mean outright mistake (MAE), root mean squared blunder (RMSE), and coefficient of assurance (R2). The MAE, RMSE, and R2 esteems are $0.926,1.422$, and 0.970 separately for engine UPDRS. These qualities are 1.334, 2.221, and 0.956 separately for Total-UPDRS. Tiwari et al. [66] proposed PD discovery model by separating cepstral highlights from the voice signs and order by help vector machine. To characterize the extricated highlights, the model utilized dimensionality decrease through direct discriminant examination and done the order by help vector machine. The proposed model is approved and tried with ten diverse AI models. The proposed technique delivered territory under the bend (AUC) of $88 \%$, affectability of $73.33 \%$, particularity of $84 \%$ and additionally, the proposed clever framework was recreated utilizing openly accessible various sorts of voice information base.

Papapetropoulos et al. [67] proposed a machine learning model to classify the person is having Parkinson's disease or not. The six classification algorithms are used for the classification of PD from the UCI Parkinson's disease Dataset. The algorithms such as logistic regression, support vector machine (SVM), Decision tree, k-nearest neighbour (KNN) and XGBOOST (Extreme gradient boosting) are used to predict the outcome whether the person is healthy or Parkinson disease effected based on the voice input parameters. The simulation results proved that the ensemble techniques produced 95\% accuracy in testing and $100 \%$ in training compared to base classification algorithm such as xgboost, bagging Classifier. Sajal et al. [68] proposed a man-made consciousness framework to separate PD patients from sound volunteers and decide various highlights of the sickness inside a partner of PD subjects. The neural network construction (NNC) strategy was utilized to group information gathered by a portable application (iMotor, Apptomics Inc., Wellesley, MA) into two classifications: PD for patients and HV. The strategy was tried on a progression of information recently gathered, and the outcomes were looked at against more conventional strategies for neural organization preparing. The NNC calculation segregated individual PD patients from HVs with 93.11\% precision and ON versus OFF state with $76.5 \%$ precision. Rastegar et al. [69] proposed a continuous observing framework with AI ways to deal with distinguish PD utilizing rest quake and vowel phonation information procured by cell phones with worked in accelerometer and voice recorder sensors. The information is principally gathered from analyzed PD patients and solid individuals for building and streamlining AI models that show better. The information is essentially gathered from analyzed PD patients and sound individuals for building and enhancing AI models that display better. The information from recently speculated PD patients is gathered, and the prepared calculations are assessed to recognize PD. After discovery PD recognized patients are associated with a close by nervous system specialist for meeting. the framework may refresh the model by retraining utilizing the most recent information subsequent to accepting patients' criticism analyzed by the nervous system specialist. The most elevated exactness in PD location utilizing disconnected information was $98.3 \%$ from voice information and $98.5 \%$ from quake information when utilized independently. In the two cases, k-closest neighbors $(\mathrm{kNN})$ gave the most noteworthy precision over help support vector machine (SVM) and innocent naïve Bayes (NB). The normal exactness of PD discovery becomes $99.8 \%$ when gathering averaging was performed on dominant part vote from $\mathrm{kNN}, \mathrm{SVM}$, and NB.

Borzì et al. [70] proposed a framework with longitudinally evaluated fringe provocative cytokines and utilized AI calculations to decide the relative degree to which the cytokines add to the longitudinal forecast of PD symptomology. It utilized serum tests from a clinically all around portrayed longitudinally followed Michael J Fox Foundation associate of PD patients with and without the regular leucine-rich recurrent kinase 2 (LRRK2) G2019S transformation. The proposed framework is utilized to assess the changeability of fringe fiery cytokine levels in people over a 1-year time period. Various components may add to the guideline of fringe cytokine levels, and while longitudinal investigations have been directed for some neurodegenerative illnesses, like Alzheimer's infection, longitudinal appraisal of fringe provocative cytokines in PD is deficient. Utilizing the standardized root mean square mistake (NRMSE) as a proportion of execution, the best expectation models were for the engine indication seriousness scales, with NRMSE of 0.1123 for the Hoehn and Yahr scale and 0.1193 for the brought together Parkinson's infection rating scale section three (UPDRS III). Yulianti et al. [71] proposed a FOG location calculation utilizing wearable frameworks and ML based order calculations. The framework got the common corruption of the strolling design going before FOG scenes, to accomplish dependable FOG expectation utilizing AI calculations and confirm whether dopaminergic treatment influences the capacity of our framework to identify and foresee FOG. An associate of 11 Parkinson's illness patients getting (on) and not accepting (off) dopaminergic treatment was furnished with two inertial sensors set on each shin, and requested to play out a coordinated up and go test. The progression to-step division is performed on the precise speed signals and ensuing element 
extraction from both time and recurrence spaces. The proposed framework utilized a covering approach for include choice and streamlined distinctive AI classifiers to get FOG and pre-FOG scenes. For pre-FOG discovery, the arrangement calculation accomplished $84.1 \%(85.5 \%)$ affectability, 85.9\% (86.3\%) particularity and $85.5 \%(86.1 \%)$ exactness. At the point when the order model was prepared with information from patients on (off) and tried on patients off (on), the ML based characterization calculation created 84.0\% (56.6\%) affectability, $88.3 \%(92.5 \%)$ explicitness and $87.4 \%(86.3 \%)$ exactness.

Yulianti et al. [71] proposed a component choice method to anticipate the PD proficiently since the dataset with numerous highlights can expand intricacy, yet not all highlights affect the consequences of the investigation. Immaterial highlights decreased the model execution. The voice dataset from UCI archive is utilized in this model. The dataset is grouped utilizing Decision Tree arrangement technique. In light of the reproduction the choice tree gained $64.17 \%$ precision and 0.6417 AUC, Decision tree with forward determination created $71.74 \%$ of exactness and 0.7171 AUC and Decision tree with in reverse choice delivered $69.42 \%$ of precision and 0.6942 AUC.

\section{COMPARISON CHART}

It is observed from the Table 1 (see Appendix), many authors have been proposed system to improve the prediction accuracy of Parkinson's disease. Several aspects of PD biomarkers need to be investigated further. More longitudinal research is required to assess which biomarker strategies would be most effective in detecting PD earlier and recognising preclinical, at-risk populations. Instead of relying solely on clinical assessment, potential diagnostic imaging or biofluid assays should be compared to the gold standard in neuropathology, since diagnostic accuracy in some settings may be poor. Methodologies for biomarker studies must be fine-tuned and standardised, which is particularly important for biofluid assays and neuroimaging protocols, as methodological variations may lead to inconsistent results. Last but not least, new biomarkers and analytical techniques must be discovered. The research of imaging methods to detect $\alpha-$ synuclein in the brain is underway. Multimodal datasets, such as those derived from various types of neuroimaging in combination with biofluid measurements and other data, can help us better understand PD and, through various models, differentiate PD patients (or at-risk persons) from healthy controls, atypical parkinsonian syndromes, and other neurodegenerative conditions. It's important to understand that sensitivity and precision coexist in a healthy way. Increased sensitivity-the ability to accurately recognise individuals who have the disease-is normally accompanied by a decrease in specificity (meaning more false-positives). Similarly, high specificity-when a test does a decent job of excluding people who don't have the diseasetypically means lower sensitivity (more false-negatives).

\section{CONCLUSION}

The similar investigation on ML techniques as far as different execution measurements like particularity, affectability, exactness, ROC, highlight chose and accuracy have been finished. From this investigation, it is noticed that for expectation, discourse and voice has been considered as a significant boundary and have delivered extensive precision. Notwithstanding discourse and voice, utilization of additional ascribes like stances, developments, outward appearances can give better outcomes and increment the exactness in diagnosing Parkinson's illness and furthermore to assist the clinical administration with higher outcomes to diminish the danger level.

\section{APPENDIX}

Table 1. Performance analysis of various existing systems

\begin{tabular}{|c|c|c|c|}
\hline $\begin{array}{l}\text { Proposed } \\
\text { Algorithm }\end{array}$ & $\begin{array}{l}\text { Features and } \\
\text { dataset used }\end{array}$ & $\begin{array}{l}\text { Number of features selected } \\
\text { for processing }\end{array}$ & Accuracy $(\%)$ \\
\hline $\begin{array}{l}\text { Minimum Redundancy } \\
\text { Maximum Relevance Feature } \\
\text { selection }\end{array}$ & Speech & 20 & 90.3 \\
\hline $\begin{array}{l}\text { Fuzzy C means Clustering and } \\
\text { Pattern Recognition }\end{array}$ & Speech & & 68.04 \\
\hline $\begin{array}{l}\text { Wrapper Feature Selection } \\
\text { Scheme }\end{array}$ & & & 72 to 92 \\
\hline Speech Signal Processing & Dysphonia & 10 & 99 \\
\hline Aritificial Neural Network & Speech & & 85.92 \\
\hline $\begin{array}{l}\text { Multilayer perception (MLP) } \\
\text { with Back Propagation Learning }\end{array}$ & & & 91.45 \\
\hline
\end{tabular}


Table 1. Performance analysis of various existing systems (continue)

\begin{tabular}{|c|c|c|c|}
\hline $\begin{array}{c}\text { Proposed } \\
\text { Algorithm }\end{array}$ & $\begin{array}{l}\text { Features and } \\
\text { dataset used }\end{array}$ & $\begin{array}{l}\text { Number of features } \\
\text { selected for processing }\end{array}$ & Accuracy $(\%)$ \\
\hline Rough Set Approach & Speech & 22 & 100 \\
\hline Genetic Algorithm and SVM & Speech & 14 & $\begin{array}{l}96.06 \text { for } 4 \text { optimized } \\
\text { features } 93.58 \text { for } 7 \text { optimized } \\
\text { features } \\
93.61 \text { for } 9 \text { optimized features }\end{array}$ \\
\hline Nested- RF and Nested-SVM & cancer & 5 & Up to 93 \\
\hline $\begin{array}{l}\text { SVM and Correlation } \\
\text { based classification }\end{array}$ & Speech & 5 & 90.5 recognition rate $0.97 \mathrm{AUC}$ \\
\hline $\begin{array}{l}\text { Neural networks, Data mining, } \\
\text { Neural analysis and } \\
\text { regression analysis }\end{array}$ & SAS Software & & $92.9,84.3,88.6,84.3$ \\
\hline $\begin{array}{l}\text { Parallel distributed } \\
\text { neural network }\end{array}$ & Voice & & 92.9 \\
\hline PCA and DLA & vision & 1 & \\
\hline SPM & Voice & 3 & 85 \\
\hline MFCC & Speech & 20 & $\begin{array}{l}\text { Quantization Result } \\
90 \text { and } 95\end{array}$ \\
\hline $\begin{array}{l}\text { Regression, Decision tree and } \\
\text { Neural network }\end{array}$ & databank & & $\begin{array}{l}\text { Error probability } \\
5.15,8.47,23.7\end{array}$ \\
\hline SVM AND RFBNN & Voice & 7 & RFBNN - 91 SVM - 83 \\
\hline FKNN based System & Voice & 2 & 96.07 \\
\hline Data mining and Machine Learning & hip & 2 & 92 \\
\hline Naive Bayes FNSB, CFS-NB & & 4 & 97 \\
\hline $\begin{array}{l}\text { SVM, KNN, PLS AND Random } \\
\text { Tree }\end{array}$ & Voice & 3 & \\
\hline Nested - RF and nested - SVM & & 5 & 93 \\
\hline $\begin{array}{l}\text { Prediction Model Tree based } \\
\text { classification }\end{array}$ & Voice & 6 & $\begin{array}{l}\text { Decision Tree - 85.08, ID3 - 75.33, } \\
\text { Decision Stumps - 83.5 }\end{array}$ \\
\hline $\begin{array}{l}\text { Neural networks (ANN),Data } \\
\text { mining Neural, Regression and } \\
\text { Decision Tree }\end{array}$ & Voice & 10 & 96.88 \\
\hline $\begin{array}{l}\text { Clonclas and Probabilistic Neural } \\
\text { Network }\end{array}$ & Immunity & 3 & 100 \\
\hline $\begin{array}{l}\text { Kernal Based extreme learning } \\
\text { Machine with Subtractive }\end{array}$ & Voice & 10 & 99.49 \\
\hline Clustering Features (SCFWKELM) & & & \\
\hline Kernal Based extreme learning & Voice & 10 & 99.49 \\
\hline $\begin{array}{lrr}\text { Machine } & \text { with } & \text { Subtractive } \\
\text { Clustering } & \text { Features } & \text { Weighting } \\
(\text { CFWKELM) } & & \end{array}$ & & & \\
\hline $\begin{array}{l}\text { SVM,KNN,Random forest and } \\
\text { Naive Bayes }\end{array}$ & Voice & 26 & 88.9 \\
\hline SVM & Eyes & 1 & 85.48 \\
\hline SVM - L2 norm Metric conjunction & Eyes & & 77 \\
\hline CART,SCM and ANN & Voice & 1 & 93.7 \\
\hline $\begin{array}{l}\text { Discretization method, SVM, C4.5, } \\
\text { k-nearest neighbour and Naive } \\
\text { Bayes }\end{array}$ & Body movement & 28 & \\
\hline Artificial Neural Network & $\begin{array}{l}\text { Neurodegerative } \\
\text { disorders }\end{array}$ & 6 & \\
\hline $\begin{array}{l}\text { Multi Layer Perception with back } \\
\text { Propagation learning Algorithm, } \\
\text { RBF, ANN }\end{array}$ & Voice & & MLP - 93.22 RBF - 86.44 \\
\hline $\begin{array}{l}\text { Random Forest(RF), SVM, GA-RF, } \\
\text { GA-SVM }\end{array}$ & Voice & 4 & 94 \\
\hline $\begin{array}{l}\text { Radial Basis Function Neural } \\
\text { Network(RBFNN) based on PSO } \\
\text { and PCA with LPF }\end{array}$ & Brain & & $\begin{array}{l}\text { RFBNN }-89.91, \mathrm{PCA}+\mathrm{RBFNN}- \\
88.92, \mathrm{PCA}+\mathrm{PSO}+\mathrm{RBFNN}- \\
88.92\end{array}$ \\
\hline $\begin{array}{l}\text { Fuzzy Entropy based feature } \\
\text { selection }\end{array}$ & Voice & & Mean Accuracy - 85.03 \\
\hline Wavelet Analysis (using MNN) & Speech & & Between 80 and 100 \\
\hline $\begin{array}{l}\text { Computer- Aided diagnosis(CADs) } \\
\text { with CFS Algorithm }\end{array}$ & & & 74.47 \\
\hline Naive Bayes and KNN & Speech & & $\begin{array}{l}\text { K-Nearest Neighbour - } 80 \text { Naïve } \\
\text { Bayes - } 93.3\end{array}$ \\
\hline
\end{tabular}




\section{REFERENCES}

[1] Armañanzas, R., Bielza, C., Chaudhuri, K. R., Martinez-Martin, P., and Pedro Larrañaga, "Unveiling relevant nonmotor Parkinson's disease severity symptoms using machine learning approach," Elsevier Journal of Artificial intelligence in medicine, vol. 58, no. 3, pp.195-202, 2013, doi: 10.1016/j.artmed.2013.04.002.

[2] Zhennao Cai, et al., "An Intelligent Parkinson's Disease Diagnostic System Based on a Chaotic Bacterial Foraging Optimization Enhanced Fuzzy KNN Approach," Computational and Mathematical Methods in Medicine, vol. 2018, Article ID 2396952, 24 pages, 2018, doi: 10.1155/2018/2396952.

[3] Salhi L., Mourad T., and Cherif A., "Voice disorders identification using multilayer neural network," Pathology, vol. 2, no. 7, pp. 8, 2008.

[4] Gan-Or Z., et al., "Differential effects of severe vs mild GBA mutations on Parkinson disease," Neurology, vol. 84, no. 9, pp. 880-887, 2015, doi: 10.1212/wnl.0000000000001315.

[5] Tarigoppula V. S Sriram, M. Venkateswara Rao, G V Satya Narayana, DSVGK Kaladhar, and T Pandu Ranga Vital, "Intelligent Parkinson Disease Prediction Using Machine Learning Algorithms," International Journal of Engineering and Innovative Technology (IJEIT), vol. 3, no. 3, 2013.

[6] Nguyen, H., Lebel, K., Bogard, S., Goubault, E., Boissy, P., and Duval C., "Using Inertial Sensors to Automatically Detect and Segment Activities of Daily Living in People With Parkinson's Disease," IEEE Transactions on Neural Systems and Rehabilitation Engineering, vol. 26, no. 1, January 2018, doi: 10.1109/TNSRE.2017.2745418.

[7] Bruno Andò, et al., "A Wearable Device to Support the Pull Test for Postural Instability Assessment in Parkinson's Disease," IEEE Transactions on Instrumentation and Measurement, vol. 67, no. 1, January 2018, doi: 10.1109/TIM.2017.2753478.

[8] Fung, A., Lai, E. C., and Lee, B., "Usability and Validation of the Smarter Balance System: An Unsupervised Dynamic Balance Exercises System for Individuals with Parkinson's Disease," IEEE Transactions On Neural Systems And Rehabilitation Engineering, vol. 26, no. 4, April 2018, doi: 10.1109/tnsre.2018.2808139.

[9] Hadjahmadi A. H. and Askari, T. J., "A Decision Support System for Parkinson's Disease Diagnosis using Classification and Regression Tree," The Journal of Mathematics and Computer Science, vol. 4, no. 2, 2012, pp. 257-263, doi: 10.22436/jmcs.04.02.15.

[10] Ali Saad, et al., "A Preliminary Study of the Causality of Freezing of Gait for Parkinson's Disease Patients: Bayesian Belief Network Approach," International Journal of Computer Science Issues, vol. 10, issue. 3, no. 2, pp.88-95, 2013.

[11] Amit S., Ashutosh M., Bhattacharya A., and Revilla F., "Understanding Postural Response of Parkinson's Subjects Using Nonlinear Dynamics and Support Vector Machines," Austin J. Biomed Eng., vol. 1, no. 1, id1005, 2014, March.

[12] Sharma, A. and Nivas Giri, R. N., "Automatic Recognition of Parkinson's disease via Artificial Neural Network and Support Vector Machine," International Journal of Innovative Technology and Exploring Engineering, vol. 4, no. 3, pp. 35-41, 2014.

[13] Tiwari, A. K., "Machine Learning based Approaches for Prediction of parkinson's disease," International Journal of Machine Learning and Applications, vol. 3, no. 2, pp. 33-39, June 2016.

[14] Tsanas, A., Little, M. A., McSharry, P. E., Spielman, J., and Ramig, L. O., "Novel speech signal processing algorithms for high accuracy classification of Parkinson's disease," IEEE Transactions on Biomedical Engineering, vol. 59, no. 5, pp.1264-1271, 2012, doi: 10.1109/TBME.2012.2183367.

[15] Chen, A. H. and Lin, C. H., "Optimizing the performance of disease classification using nested-random forest and nested-support vector machine classifiers," Journal of Chemical and Pharmaceutical Research, vol. 5, no. 12, pp. 1521-1528, 2013.

[16] Azad, C., Jain, S., and Jha, V. K., "Design and Analysis of Data Mining Based Prediction Model forParkinson's disease," vol. 1, no. 1, 181-189, 2014.

[17] Bouchikhi S., Boublenza, A., Benosman, A., and Chikh, M. A., "Parkinson's disease Detection with SVM classifier and Relief-F Features Selection Algorithm," South East Europe Journal of Soft Computing, vol. 2, no. 1, 2013.

[18] Chen, A. H., Cheng, C. H., and Lin, C. H., "The Improvement of Parkinson's Disease Classification using Genetic Algorithm- Random Forests and Genetic Algorithm-Support Vector Machine Methods," International Journal of Advancements in Computing Technology, vol. 4, no. 21, pp. 320-328, 2012, doi: 10.4156/ijact.vol4.issue21.37.

[19] Chen, A. H., and Lin, C. H., "Optimizing the performance of disease classification using nested-random forest and nested support vector machine classifiers," Journal of Chemical \& Pharmaceutical Research, vol. 5, no. 12, pp. 1521-1528, 2013.

[20] Chen, H. L., Huang, C. C., Yu, X. G., Xu, X., Sun, X., Wang, G., and Wang, S. J., "An efficient diagnosis system for detection of Parkinson's disease using fuzzy k-nearest neighbour approach," Expert systems with applications, vol. 40, no. 1, pp. 263-271, 2013, doi: 10.1016/j.eswa.2012.07.014.

[21] Cho, C., Wen-Hung Chao, Sheng-Huang Lin, and You-Yin Chen, "A vision-based analysis system for gait recognition in patients with Parkinson's disease," Elsevier Journal of Expert Systems with Applications, vol. 36, no. 3, pp. 7033-7039, 2009, doi: 10.1016/j.eswa.2008.08.076.

[22] Farhad, S. G. and Peyman, M., "A Case Study of Parkinson's disease Diagnosis using Artificial Neural Networks," International Journal of Computer Applications, vol. 73, no.19, pp. 1-6, 2013, doi: 10.5120/12990-9206.

[23] Wu, D., Warwick, K., Ma, Z., Gasson, M. N., Burgess, J. G., Pan, S., and Aziz, T. Z., "Prediction of Parkinson's disease tremor onset using a radial basis function neural network based on particle swarm optimization," International journal of neural systems, vol. 20, no. 2, pp. 109-116, 2010, doi: 10.1142/s0129065710002292. 
[24] Hazan, H., Hilu, D., Manevitz, L., Ramig, L. O., and Sapir, S., "Early diagnosis of Parkinson's disease via machine learning on speech data," In Electrical \& Electronics Engineers in Israel (IEEEI), 2012 IEEE 27th Convention of. IEEE, pp. 1-4, 2012, November.

[25] Nguyen, H., Lebel, K., Bogard, S., Goubault, E., Boissy, P., and Duva, C., "Using Inertial Sensors to Automatically Detect and Segment Activities of Daily Living in People With Parkinson's Disease," IEEE Transactions on Neural Systems and Rehabilitation Engineering, vol. 26, no. 1, pp. 197-204, Jan 2018, doi: 10.1109/TNSRE.2017.2745418.

[26] Rustempasic I., and Can, M., "Diagnosis of Parkinson 's disease using Fuzzy C-Means Clustering and Pattern Recognition," South East Europe Journal of Soft Computing, vol. 2, no. 1, pp. 42-49, 2013.

[27] Rusz J., et al., "Acoustic analysis of voice and speech characteristics in early untreated Parkinson's disease," 7th International Workshop on Models and Analysis of Vocal Emissions for Biomedical Applications, 2011, pp. 181184.

[28] Kapoor, T., and Sharma, R. K., "Parkinson's disease Diagnosis using Mel-frequency Cepstral Coefficients and Vector Quantization," Int. Journal of Computer Applications, vol. 14, no. 3, pp. 43-46, 2011, doi: 10.5120/18212393.

[29] Kaya, E., Findik, O., Babaoglu, I., and Arslan, A, "Effect of discretization method on the diagnosis of Parkinson's disease," International Journal of Innov. Comp. Inf. Control, vol. 7, no. 8, pp. 4669-4678, 2011.

[30] Revett, K., Gorunescu, F., and Salem, A. M., "Feature selection in Parkinson's disease: A rough sets approach," IEEE International Multiconference on In Computer Science and Information Technology (IMCSIT'09), October 2009 , pp. 425-428, doi: 10.1109/IMCSIT.2009.5352688.

[31] Khemphila, A. and Boonjing, V., "Parkinsons disease classification using neural network and feature selection," World Academy of Science, Engineering and Technology, International Journal of Mathematical, Computational, Physical, Electrical and Computer Engineering, vol. 6, no. 4, pp. 377-380, 2012, doi: 10.5281/zenodo.1071075.

[32] Kihel, B. K., and Benyettou, M., "Parkinson's disease recognition using artificial immune system," Journal of Software Engineering and Applications, vol. 4, no. 07, p. 391, 2011, doi: 10.4236/jsea.2011.47045.

[33] Rouhollahi, K., Andani, M. E., Karbassi, S. M., and Izadi, I., "Design of robust adaptive controller and feedback error learning for rehabilitation in Parkinson's disease: a simulation study," IET Journal of System Biology, vol. 11, no. 1, pp. 19-29, March 2017, doi:10.1049/iet-syb.2016.0014.

[34] Luukka, P., "Feature selection using fuzzy entropy measures with similarity classifier," Expert Systems with Applications, vol. 38, no. 4, pp. 4600-4607, 2011, doi: 10.1016/j.eswa.2010.09.133.

[35] Ma, C., Ouyang, J., Chen, H. L., and Zhao, X. H., "An Efficient Diagnosis System for Parkinson's Disease Using Kernel-Based Extreme Learning Machine with Subtractive Clustering Features Weighting Approach," Computational and mathematical methods in medicine, 2014, doi: 10.1155/2014/985789.

[36] Can, M., "Diagnosis of Parkinson's disease by Boosted Neural Networks," South East Europe Journal of Soft Computing, vol. 2, no. 1, pp. 7-13, March 2013, doi: 10.21533/scjournal.v2i1.38.

[37] Caglar, M. F., Cetisli, B., and Toprak, I. B., "Automatic recognition of Parkinson's disease from sustained phonation tests using ANN and adaptive neuro-fuzzy classifier," J. of Eng. Science and Design, vol. 1, no. 2, pp. 59-64, 2010.

[38] Shahbakhi, M., Far, D. T., and Tahami, E., "Speech Analysis for Diagnosis of Parkinson's Disease Using Genetic Algorithm and Support Vector Machine," Journal of Biomedical Science and Engineering, vol. 7, no. 04, pp. 147-156, 2014, doi: 10.4236/jbise.2014.74019.

[39] Morales, D. A., et al., "Predicting dementia development in Parkinson's disease using Bayesian network classifiers," Psychiatry Research: NeuroImaging, vol. 213, no. 2, pp. 92-98, 2013, doi:10.1016/j.pscychresns.2012.06.001.

[40] Ramani, R. G., and Sivagami, G., "Parkinson Disease Classification using Data Mining Algorithms," International Journal of Computer Applications, vol. 32, no. 9, pp. 17-22, 2011, doi: 10.5120/3932-5571.

[41] Nivedita, C., Yogender, A., and Sinha, R. K., "Artificial Neural Network based Classification of Neurodegenerative Diseases," Advances in Biomedical Engineering Research (ABER), vol. 1, no. 1, 2013.

[42] Ozcift, A. and Gulten, A., "Classifier ensemble construction with rotation forest to improve medical diagnosis performance of machine learning algorithms," Computer methods and programs in biomedicine, vol. 104, no. 3, pp. 443-451, 2011, doi: 10.1016/j.cmpb.2011.03.018.

[43] Prashanth, R., Roy, S. D., Mandal, P. K., and Ghosh, S., "Parkinson's disease detection using olfactory loss and REM sleep disorder features," In Engineering in Medicine and Biology Society (EMBC), 2014 36th Annual International Conference of the IEEE, pp. 5764-5767. IEEE, 2014, August, doi: 10.1109/embc.2014.6944937.

[44] Przybyszewski, A. W., "Applying Data Mining and Machine Learning Algorithms to predict symptom development in Parkinson's disease," In Annales Academiae Medicae Silesiensis, vol. 68, no. 5, pp. 332-349, 2014.

[45] Das, R., "A comparison of multiple classification methods for diagnosis of Parkinson disease," Elsevier Journal of Expert Systems with Applications, vol. 37, no. 2, pp.1568-1572, 2010, doi: 10.1016/j.eswa.2009.06.040.

[46] Alcalay, R. N., et al., "Cognitive performance of GBA mutation carriers with early-onset PD: the CORE-PD study," Neurology, vol. 75, no. 1821, pp, 1434-1440, Mar 2012, doi: 10.1212/wnl. 0b013e318253d54b.

[47] Saad, A, Zaarour, I, Bejjani, P, and Ayache, M., "Handwriting and Speech Prototypes of Parkinson Patients: Belief Network Approach," International Journal of Computer Science Issues, vol. 9, no. 3, 2012.

[48] Salvatore, C., et al., "Machine learning on brain MRI data for differential diagnosis of Parkinson's disease and Progressive Supranuclear Palsy,” J. of Neur. Meth., pp. 230-237, 2014, doi: 10.1016/j.jneumeth.2013.11.016. 
[49] Sellam, V. and Jagadeesan, J., "Classification of Normal and Pathological Voice Using SVM and RBFNN," Journal of Signal and Information Processing, vol. 05, no. 01, 2014, doi: 10.4236/jsip.2014.51001.

[50] Stemmer, T. B. E. N. G., "Detection of persons with Parkinson's disease by acoustic, vocal, and prosodic analysis," IEEE Work. on Auto. Speech Recog. and Unders., 2011, pp. 478-483, doi: 10.1109/ASRU.2011.6163978.

[51] Tsanas, A, Little, M. A., McSharry, P. E., and Ramig, L. O., "Nonlinear speech analysis algorithms mapped to a standard metric achieve clinically useful quantification of average Parkinson's disease symptom severity," Journal of the Royal Society Interface, vol. 8, no. 59, pp. 842-855, 2010, doi: 10.1098/rsif.2010.0456.

[52] Yang, C., Yang, Q., Xue, H., Tang, N. L. S., and Yu, W., "MegaSNPHunter: a learning approach to detect disease predisposition SNPs and high level interactions in genome wide association study," BMC Bioinformatics, vol. 10, no. 13, 2009 Jan, doi: 10.1186/1471-2105-10-13.

[53] Cai, Z., Gu, J., and Chen, H., "A New Hybrid Intelligent Framework for Predicting Parkinson's Disease," in IEEE Access, vol. 5, pp. 17188-17200, 2017, doi: 10.1109/access.2017.2741521.

[54] Mandal, I. and Sairam, N., "New machine-learning algorithms for prediction of Parkinson's disease," International Journal of Systems Science, vol. 45, no. 3, pp. 647-666, 2014, doi: 10.1080/ 00207721.2012.724114.

[55] Pahuja, G. and Nagabhushan, T. N., "A Comparative Study of Existing Machine Learning Approaches for Parkinson's Disease Detection," IETE Journal of Research, vol. 67, no. 1, pp. 4-14, 2018, doi: 10.10 80/ 03772063.2018.1531730.

[56] Rastegar, D. A., Ho, N., Halliday, G. M., and Dzamko, N., "Parkinson's progression prediction using machine learning and serum cytokines," Nat. Part. J. Parkinson's Dis., vol. 5, no. 14, pp. 1-8, 2019, doi: 10.1038/s41531019-0086-4.

[57] Mathur, R., Pathak, V., and Bandil, D., "Parkinson Disease Prediction Using Machine Learning Algorithm," Emerging Trends in Expert Applications and Security, vol. 841, pp 357-363, 2019, doi: 10.1007/978-981-13-2285$3 \_42$.

[58] Ulagamuthalvi, V., Kulanthaivel, G., Reddy, G. S. N., and Venugopal, G., "Identification of Parkinson's Disease Using Machine Learning Algorithms," Biosci. Biotec. Res. Comm., vol. 13, no. 2, 2020, doi: $10.21786 / \mathrm{bbrc} / 13.2 / 32$.

[59] Ulagamuthalvi, S. K. V., "A Comparative Study on Tumor Classification,” Research Journal of Pharmacy and Technology, vol. 12, no. 1, pp. 407-411, 2019, doi: 10.5958/0974-360x.2019.00074.x.

[60] Ulagamuthalvi, S, K. V., "Fusion of feature selection and Random Forest for an Anomaly based intrusion detection system," J. of Comp. and Theoretical Nan science, vol. 16, pp. 3603-3607, 2019, doi: 10.1166/jctn.2019.8332.

[61] Viswanathan, M. B. R., and Naib, B. B., "Feedback-based gait identification using deep neural network classification," Journal of Critical Reviews, vol. 7, pp. 661-667, 2020, doi: 10.31838/jcr.07.04.125.

[62] Mozhdehfarahbakhsh, A., Chitsazian, S., Chakrabarti, P., Chakrabarti, T., Kateb, B., and Nami, M., "An MRIbased Deep Learning Model to Predict Parkinson's Disease Stages," Journal of Critical Reviews, preprint from medRxiv, February 2021, doi: 10.1101/2021. 02.19.21252081.

[63] Makarious, M. B., et al., "Multi-Modality Machine Learning Predicting Parkinson's Disease," preprint from bioRXiv, March 2021, doi: 10.1101/2021.03.05.434104.

[64] Shahid, A. H. and Singh, M. P., "A Deep Learning Approach for prediction of Parkinson's Disease progression," Biomedical Engineering Letters, vol. 10, pp. 227-239, 2020, doi: 10.1007/s13534-020-00156-7.

[65] Rahman, A., Rizvi, S. S., Khan, A., Abbasi, A. A., Khan, S. U., and Chung, T., "Parkinson's Disease Diagnosis in Cepstral Domain Using MFCC and Dimensionality Reduction with SVM Classifier," Mobile Information Systems, vol 2021, pp. 1-10, March 2021, doi: 10.1155/2021/8822069.

[66] Tiwari, H., Shridhar, S. K., Patil, P. V., Sinchana, K. R., and Aishwarya, G., "Early Prediction of Parkinson's Disease using Machine Learning and Deep Learning Approaches”, EasyChair preprint, no. 4889, January 2021.

[67] Tsoulos, I. G., Mitsi, G., Stavrakoudis A., and Papapetropoulos S., "Application of Machine Learning in a Parkinson's Disease Digital Biomarker Dataset Using Neural Network Construction (NNC) Methodology Discriminates Patient Motor Status", Front. ICT, vol. 6, no. 10, 2019, doi: 10.3389/fict.2019.00010.

[68] Sajal, M. S. R., Ehsan, M. T., Vaidyanathan, R., Wang, S., Aziz, T., and Mamun, K. A. A., "Telemonitoring Parkinson's Disease using Machine Learning by combining tremor and voice analysis," Brain Informatics, vol. 7, no. 12 , pp. 1-11, 2020, doi: 10.1186/s40708-020-00113-1.

[69] Rastegar, D. A., Ho, N., Halliday, G. M., and Dzamko, N., "Parkinson's progression prediction using machine learning and serum cytokines," Springer Nature Journal of Parkinson's Disease, vol. 5, no. 14, July 2019, doi: 10.1038/s41531-019-0086-4.

[70] Borzì, L., Mazzetta, I., Zampogna, A., Suppa, A., Olmo, G., and Irrera, F, "Prediction of Freezing of Gait in Parkinson's Disease Using Wearables and Machine Learning," Sensors 2021, vol. 21, no. 2, p. 614, doi: $10.3390 / \mathrm{s} 21020614$.

[71] Yulianti, Syapariyah, A. N., Saifudin, A., and Desyani, T., "Feature Selection Techniques to Choose the Best Features for Parkinsons Disease Predictions Based on Decision Tree," ICComSET 2019, Journal of Physics: Conference Series, IOP Publishing, vol. 1477, no. 3, 2020, doi: 10.1088/1742-6596/1477/3/032008. 


\section{BIOGRAPHIES OF AUTHORS}
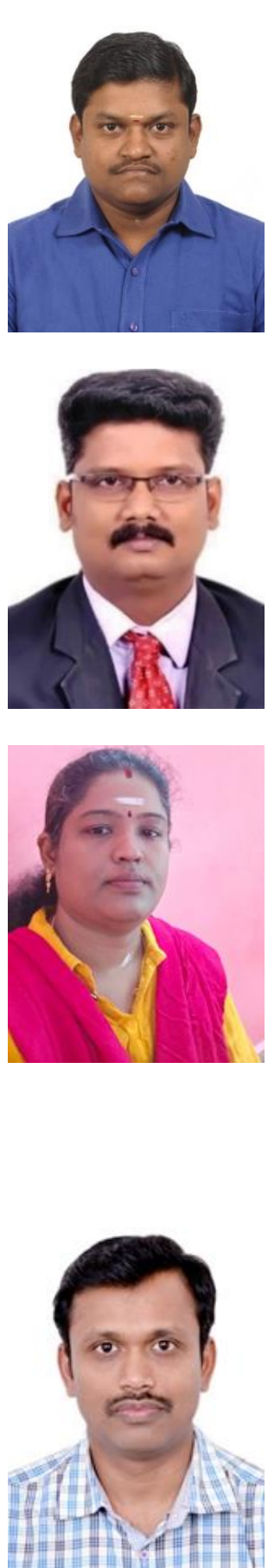

N. Prasath has obtained his Doctoral Degree from Anna University, Chennai at 2017 and Master of Technology from SASTRA University at 2009 and Undergraduate degree under Anna University Chennai in 2006. He is having 13+ years of experience in teaching and Industry. He has published 4 patents and published more than 15 research papers in refereed conferences and in journals. He is a member many professional societies. His research interests include MANET, Sensor Networks, IoT and Cloud, Cyber Physical System and Machine Learning.

Vigneshwaran Pandi has obtained his Doctoral Degree in Anna University Chennai in 2016 and Master of Engineering under Anna University Chennai in June 2005. He is having 19.2 years of experience and specialization in Cybersecurity. Presently, He is working as Associate Professor at the SRM Institute of Science and Technology, Chennai. He has published more than 18 papers in various international journals and 5 in International Conferences. His area of interest includes Security, Routing, and Intelligent Data Analysis.

Sindhuja Manickavasagam in the Department of Information Technology, Rajalakshmi Engineering College from August 2006 onwards. She is having more than 15 years of experience in teaching. Presently she is acting as Assistant Professor (Senior Grade). She is pursuing Doctoral Program under Anna University in the field of Data Analytics. Her research interest includes Artificial Intelligence, Machine Learning, Deep Learning, Bigdata Analytics, and Bioinformatics. She has published 14 international Journals including 3 from SCI and Scopus Indexed Journals. She has published one patent titled "Arm Band for Blood Testing" under the category of Design patent. She has visited Japan and Malaysia for presenting her research work in reputed International Conferences. Presently, she is working in the project of Cancer Analysis. She is a Certified Talend DI developer by Virtusa, IBM certified DB2 and Tivoli developer. She acted as resource person for various workshops held in other Engineering Colleges and University on her research topics.

Prabu Ramadoss is a well experienced IT Systems Architect and currently working as "Senior Solution Architect" at Dassault Systèmes (Australia) which is one among largest global software companies that develops software for 3D product design, simulation, and manufacturing. In his past, he briefly worked as Enterprise Architect for General Electric (GE) and Boeing. He completed his bachelor of technology in Biotechnology from Bharathidasan University, India in 2005 and obtained his Engineering Master Degree in Computer Science from SASTRA University, India in 2007. His area of interests are advanced 3D Design and Simulation technologies for Industry 4.0 Revolution. 